\title{
Estudo do impacto de diferentes índices associados ao El Niño Oscilação Sul na produtividade de soja no Rio Grande do Sul
}

\section{Study of the impact of different indices associated with El Niño Southern Oscillation on soybean yield in Rio Grande do Sul}

\author{
Diogo Alessandro Arsego ${ }^{1}$, Simone Erotildes Teleginski Ferraz ${ }^{1}$, Nereu Augusto Streck ${ }^{1}$, \\ Andrea de Oliveira Cardoso ${ }^{2}$ e Alencar Junior Zanon ${ }^{1}$ \\ ${ }^{1}$ Universidade Federal de Santa Maria, Santa Maria, RS, Brasil \\ ${ }^{2}$ Univesidade Federal do ABC, SP, Brasil
}

\section{Resumo}

O principal fator associado a queda de produtividade da soja no Rio Grande do Sul (RS) é a distribuição irregular da precipitação sendo o El Niño Oscilação Sul (ENOS) o maior responsável pela variabilidade na precipitação. O objetivo deste trabalho foi analisar a relação entre indicadores climáticos associados ao ENOS e a produtividade de soja no Estado. Foram avaliadas séries de produtividade de soja de 87 municípios entre 1984 e 2013. Estas séries foram ordenadas em três grupos de comportamento semelhante (alta, média e baixa produtividade). Os perfis médios destes grupos foram correlacionados com anomalias de Temperatura da Superfície do Mar na região do Niño 3.4, o Índice de Oscilação Sul (IOS) e o Multivariate ENSO Index (MEI) com diferentes defasagens. O IOS e o MEI apresentaram correlações mais elevadas com a produtividade em períodos de maior necessidade hídrica da cultura. Episódios de El Niño estiveram associados a elevação de produtividade e em situações de neutralidade e La Niña a influência do fenômeno é menos evidente. Além disso, a ausência de uma correlação significativa na época de preparação de safra e início do plantio evidencia a necessidade da inclusão de novos índices nas discussões de planejamento agrícola no Estado.

Palavras-chave: variabilidade interanual, produtividade de soja, El Niño Oscilação Sul.

\section{Abstract}

The main factor associated with decreaes in soybean yield in Rio Grande do Sul is the irregular distribution of precipitation and the El Niño Southern Oscillation (ENSO) is the major responsible for the precipitation variability. The aim of this work was to analyze the relationship between climatic indicators associated with ENSO and soybean yield in the State. Series of soybean yield from 87 municipalities were evaluated between 1984 and 2013. These series were ordered in three groups of similar behavior (high, medium and low yield). The mean profiles of these groups were correlated with indices for Sea Surface Temperature anomalies in the Niño 3.4 region, the Southern Oscillation Index (SOI) and the Multivariate ENSO Index (MEI) with differents lags. SOI and MEI showed higher correlations with yield in periods of higher water requirement of the crop. El Niño episodes have been associated with increased yield and in situations of neutrality and La Niña, the influence of the phenomenon is less evident. In addition, the absence of a significant correlation in the time of preparation of the crop and in the beginning of the plantation evidences the necessity of including new indices in the discussions of agricultural planning in the State.

Keywords: interannual variability, soybean yield, El Niño Southern Oscillation. 


\section{Introdução}

A atividade agropecuária está intimamente relacionada a economia brasileira (CEPEA, 2017). No Rio Grande do Sul (RS), a maior parte da produção agrícola está associada as culturas de verão (IBGE, 2017). Neste sentido, destaca-se a soja com uma produção total de 15.700.264 toneladas no ano de 2015, sendo o RS o terceiro Estado em produção desta cultura (IBGE, 2017).

Dentre as atividades econômicas, a agricultura é a mais dependente das condições meteorológicas (Hoogenboom, 2000) e períodos de estiagem, frequentemente, estão associados a queda da produtividade e prejuízos para a economia (Lazzari, 2006). Para a soja, a precipitação compreendida entre janeiro e março é a principal variável meteorológica associada a variabilidade da produtividade sobre o RS (Berlato e Fontana, 1999). Embora o RS não tenha uma estação seca definida e possua totais anuais de precipitação elevados (1.200 a $2.000 \mathrm{~mm} /$ ano) (Reboita et al., 2010), a distribuição irregular da chuva durante a estação de crescimento se apresenta como fator limitante ao rendimento potencial da cultura (Sentelhas et al., 2015; Zanon et al., 2016).

Dada a elevada relação entre a variabilidade climática e a produtividade agrícola, a compreensão dos padrões de teleconexão responsáveis pela variabilidade sazonal da precipitação sobre o RS torna-se fundamental para o melhor planejamento de safra. Entre os padrões globais de teleconexão, o El Niño-Oscilação do Sul (ENOS) é a principal fonte de variabilidade climática interanual (Kayano e Andreoli, 1998). Estudos prévios demonstraram que fases quentes (frias) do ENOS estão associadas a elevação (diminuição) da precipitação sobre o RS (Rao e Hada, 1990) e, consequentemente, resultam em aumento (queda) da produtividade de soja no Estado gaúcho (Berlato e Fontana, 1999).

A relação entre o ENOS, precipitação e produtividade indica a possibilidade da utilização de índices associados ao fenômeno para estimativas de produtividade de soja no RS. Santos e Diniz (2014) demonstraram ser possível realizar estimativas de precipitação no RS baseadas em índices associados ao ENOS. Apesar do estudo pioneiro de Berlato e Fontana (1999) apresentar as consequências do ENOS na precipitação trimestral de janeiro a março e seu impacto na produtividade, não foi estabelecida uma relação direta entre este padrão de teleconexão e a variabilidade da produtividade de soja no Estado. Além disso, no período analisado (1976-1995) por Berlato e Fontana (1999) apenas dois eventos de La Niña foram registrados o que indica uma lacuna para estudos da influência da fase fria do fenômeno na produtividade agrícola da soja no RS.

O objetivo deste estudo é analisar o potencial de índices associados ao ENOS para estimativas de produtividade de soja no Rio Grande do Sul. Além disso a ampliação da série histórica, fornece uma relação mais detalhada entre os impactos de cada fase do fenônemo (El Niño La Niña e neutralidade) nos padrões de precipitação e produtividade de soja no Estado gaúcho.

\section{Materias e Métodos}

Neste estudo, foram utilizadas séries históricas de produtividade de soja de 497 municípios do RS entre 1984 a 2013 . Para analisar apenas séries de municípios representativos para a cultura foram aplicados dois filtros: remoção dos municípios com dados faltantes ou repetidos por três anos consecutivos e eliminação de séries com correlação inferior a 0,7 com a produtividade média do Estado.

Em séries históricas de produtividade, há uma tendência de aumento do rendimento ao longo dos anos resultante de melhorias na tecnologia agrícola (novas cultivares e melhoria das práticas de manejo de planta e solo). Desta forma, os 87 municípios restantes tiveram a tendência tecnológica de suas séries removidas com base em uma regressão linear simples (Equação 1), na qual assume-se que a tendência linear é resultado de melhoramentos genéticos e incremento em maquinário agrícola e a variação interanual está associada a variabilidade climática (Wenjiao et al., 2013).

$$
Y_{c i}=Y_{i}-Y\left(X_{i}\right)+Y\left(X_{f}\right)
$$

sendo que $Y_{c i}$ é a produtividade corrigida para o ano $i ; Y_{i}$ é a produtividade original do ano $i$; $Y\left(X_{i}\right)$ é a produtividade estimada para o ano $i$ através do modelo de regressão e $Y\left(X_{f}\right)$ é a produtividade do último ano estimada pela regressão linear.

Para separar as séries municipais em grupos de comportamento semelhante em termos de produtividade foi utilizado o método da análise de cluster baseado na medida de distância Euclidiana (Wilks, 2006). O critério utilizado para o agrupamento foi o método de Ward (1963) e foi realizado um corte no dedrograma de forma a reter três grandes grupos de produtividade (alta, média e baixa).

A utilização da análise de cluster permitiu a obtenção de uma série média de cada grupo que foi correlacionada a três diferentes índices associados ao ENOS: anomalias de Temperatura da Superfície do Mar (TSM) na região do Niño 3.4 (Nino), Índices de Oscilação Sul (IOS) e Multivariate ENSO Index (MEI), todos disponíveis em: https://www.esrl.noaa.gov/psd/data/climateindices/list/.

Assumiu-se o mês de abril como referente a colheita da soja e o mês de setembro como mês anterior ao início do plantio (Fontana et al., 2001). Assim sendo, a primeira correlação foi realizada entre a produtividade média de cada grupo e os índices referentes ao ENOS no mês de setembro, a segunda correlação entre as séries de produtividade e os índices do mês de outubro e assim, sucessivamente, até a correlação realizada entre os perfis de produtividade e os índices referentes ao ENOS do mês de abril.

Em adição as correlações, é apresentado um diagrama de dispersão referente a cada fase do ENOS (El Niño, La Niña e neutralidade) e os desvios de produtividade para os três grupos. Para esta análise, foram utilizadas as anomalias de TSM na região 
do Niño 3.4 para a separação dos eventos. Eventos de El Niño (La Niña) foram assumidos quando a anomalia da média móvel trimestral é maior ou igual (menor ou igual) a $0,5^{\circ} \mathrm{C}$ por, pelo menos, cinco trimestres consecutivos.

\section{Resultados}

A representação espacial em grupos de comportamento homogêneo de produtividade de soja dos municípios utilizados neste estudo pode ser visualizada na Figura 1(a). Nota-se, que os municípios do Grupo 1 (G1) situam-se, principalmente, no nordeste do Estado e este grupo é o que apresenta maior produtividade média ao longo do período de estudo (Figura 1(b)). Por outro lado, os membros do Grupo 3 (G3), que se concentram mais a noroeste e oeste do Estado, apresentam a menor produtividade média (Figura 1(b)). Por fim, os municípios que compõe o Grupo 2 (G2), distribuem-se entre o G1 e G2, próximo a região central do Estado e apresentam produtividade intermediária (Figura 1(b)).

As diferenças de produtividade entre os grupos pode ser explicada pelo risco de déficit hídrico. Segundo da Cunha et al. (2001), o nordeste do Rio Grande do Sul, região na qual predominam municípios do G1, possui menor risco de queda de produtividade por déficit hídrico em virtude das temperaturas mais baixas e totais pluviométricos mais elevados em comparação com os outros grupos. Por outro lado, o noroeste do RS, onde se encontra a maior parte dos municípios do G3, apresenta maior potencial de queda de produtividade em virtude do déficit hídrico (da Cunha et al., 2001).

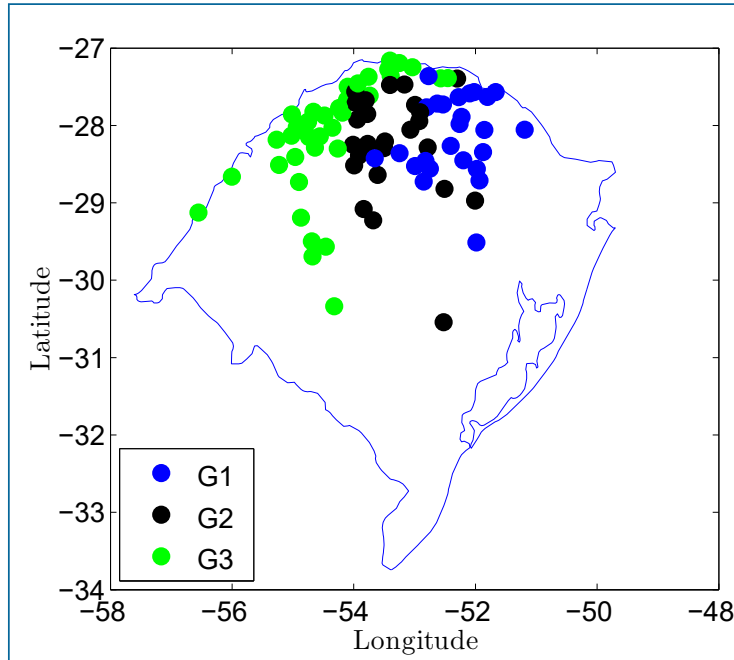

(a) Regiões homogêneas de produtividade de soja.

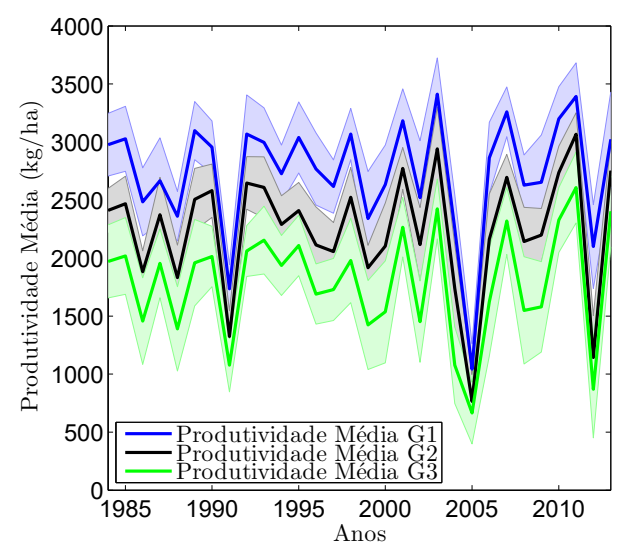

(b) Perfis médios de produtividade de soja.

Figura 1: Regiões homogêneas e perfis médios de produtividade de soja no Rio Grande do Sul resultantes da análise de agrupamento. A Figura 1(a) apresenta a distribuição espacial dos grupos G1 (azul), G2 (preto) e G3 (verde). A Figura 1(b) apresenta os perfis médios de produtividade de grãos de soja dos grupos: G1 (azul), G2 (preto) e G3 (verde). A área sombreada delimita o desvio padrão de cada uma das séries.

Na Tabela 1 estão evidenciadas as correlações entre os índices climáticos em cada mês de referência e a produtividade de soja nos grupos homogêneos. Destacam-se, inicialmente, as baixas correlações entre a região oceânica do Niño 3.4 e a produtividade de soja para os três grupos homogêneos independente da defasagem utilizada. Percebe-se também, um padrão de correlações negativas e significativas com o IOS nos meses de dezembro e janeiro com inversão do sinal a partir de fevereiro. Para o MEI, nota-se elevada correlação positiva entre dezembro e janeiro. Os resultados acima listados convergem aos estudos prévios de Berlato e Fontana (1999) e Berlato e Fontana (2003) que sinalizam que a fase quente do fenômeno está associada a elevação da produtividade de soja no Rio Grande do Sul.

A comparação entre os índices demonstra que, apesar do MEI apresentar em alguns casos correlações significativas, o IOS possui as correlações mais elevadas para os três grupos. Tais resultados sugerem a monitoramento destes índices climáticos em épocas de preparativo de safra de forma a obter melhores estimativas de produtividade. Nota-se ainda que, de uma forma geral, o G3 apresenta as correlações mais elevadas enquanto que o G1 possui valores mais baixos. Estudos prévios que analisaram a influência do ENOS na precipitação sobre a América do Sul demonstraram que, para o RS, a correlação, entre os meses de novembro e fevereiro, diminui em direção ao nordeste do Estado (Diaz et al., 1997) justificando, assim, a diferença entre os grupos. 
Tabela 1: Correlação entre a produtividade média dos três grupos homogêneos e os índices climáticos referentes ao Niño, IOS e MEI para os meses de setembro a abril. Os valores marcados com * e ** possuem nível de confiança da correlação superior a $80 \%$ e $90 \%$, respectivamente.

\begin{tabular}{|c|c|c|c|c|c|c|c|c|c|}
\hline & \multicolumn{3}{|c|}{ Nino } & \multicolumn{3}{|c|}{ IOS } & \multicolumn{3}{|c|}{ MEI } \\
\hline & G1 & G2 & G3 & G1 & G2 & G3 & G1 & G2 & G3 \\
\hline SET & $-0,043$ & 0,005 & 0,049 & 0,111 & 0,093 & 0,058 & 0,011 & 0,036 & 0,080 \\
\hline OUT & 0,003 & 0,052 & 0,099 & $-0,119$ & $-0,113$ & $-0,176 *$ & 0,041 & 0,066 & 0,122 \\
\hline NOV & 0,016 & 0,069 & 0,122 & 0,096 & 0,050 & $-0,019$ & 0,036 & 0,075 & 0,136 \\
\hline DEZ & 0,008 & 0,057 & 0,112 & $-0,090$ & $-0,164 *$ & $-0,212 * *$ & 0,046 & 0,101 & $0,154 *$ \\
\hline JAN & 0,030 & 0,080 & 0,134 & $-0,182^{*}$ & $-0,214 * *$ & $-0,217 * *$ & 0,118 & $0,164 *$ & $\mathbf{0 , 2 0 0} * *$ \\
\hline FEV & 0,014 & 0,071 & 0,122 & $0,194 *$ & $0,140 *$ & 0,052 & 0,054 & 0,105 & $0,146 *$ \\
\hline MAR & 0,016 & 0,089 & 0,133 & $-0,004$ & $-0,049$ & $-0,054$ & 0,014 & 0,068 & 0,121 \\
\hline ABR & $-0,033$ & 0,054 & 0,095 & $0,231 * *$ & $0,187 *$ & 0,139 & $-0,001$ & 0,050 & 0,072 \\
\hline
\end{tabular}

Na Figura 2 estão dispostas as anomalias de TSM na região do Niño 3.4 no mês de janeiro, mês em que as correlações foram mais elevadas, e os desvios de produtividade de soja dos três grupos homogêneos. Nota-se que, concordando com os estudos de Berlato e Fontana (1999) e Berlato e Fontana (2003), eventos de El Niño estiveram associados a elevação da produtividade (80\% dos casos). Apesar disso, destaca-se que o ano em que foi observado o maior desvio negativo de produtividade para os três grupos (2005) foi um ano sob influência da fase quente do fenômeno.

Para eventos de neutralidade, em 53\% dos casos houve queda de produtividade. Para a região central do Estado, Alberto et al. (2006) já haviam destacado que eventos de neutralidade são os mais propensos a queda de produtividade de soja em virtude do déficit hídrico.

Por outro lado, a influência negativa esperada para eventos de La Niña não fica tão clara. Em 52\% dos casos de La Niña houve queda de produtividade de soja, mas foi em um ano sob influência de La Niña (2011) que foi observado o maior desvio positivo de produtividade para os três grupos. Em estudo global sobre os efeitos do ENOS na agricultura, Lizumi et al. (2014) destacaram que, para o Sul do Brasil, há um forte impacto positivo na produção de soja na fase quente e efeito menos expressivo em anos de La Niña. Além disso, episódios frios do ENOS exercem maior impacto na precipitação da metade sul do Estado (Grimm et al., 1998), região esta que não está inserida neste estudo por ser responsável por menos de $6 \%$ da produtividade estadual de soja.

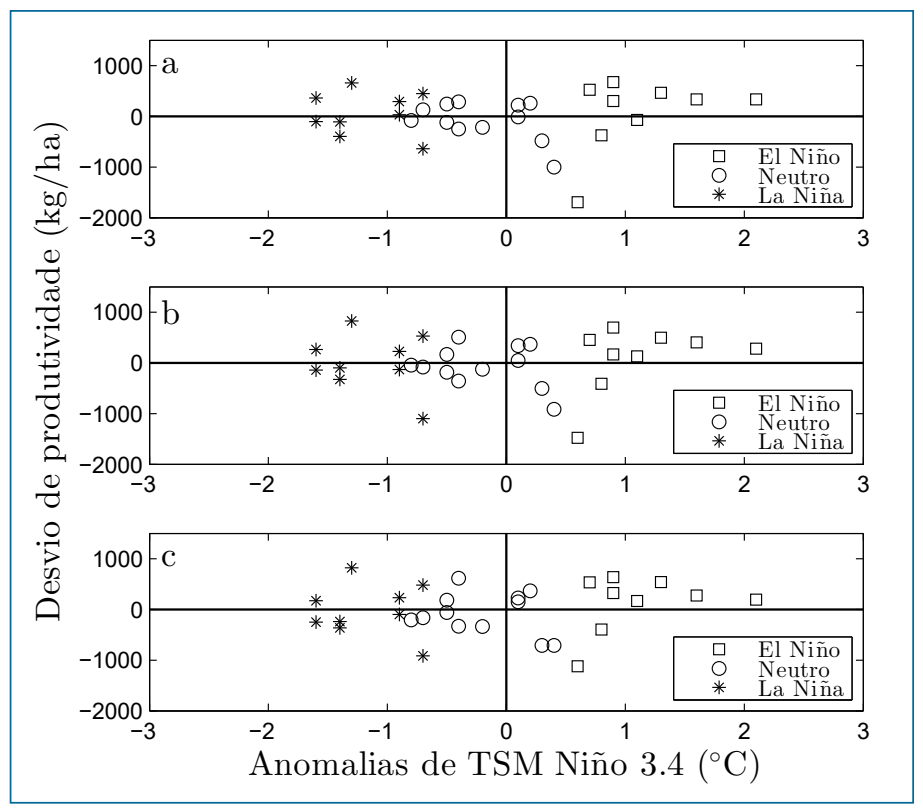

Figura 2: Relação entre anomalias de TSM na região do Niño 3.4 em janeiro e anomalias de produtividade para o grupo: a) G1, b) G2 e c) G3. No gráfico, quadrados representam eventos de El Niño, círculos de neutralidade e asteríscos de La Niña.

Estudos de precipitação no RS mostram que tanto padrões de baixa frequência, como a Oscilação Decadal do Pacífico (Kaiano e Andreoli, 2007), quanto de de alta frequência, como a Oscilação Antártica (Reboita et al., 2009), têm influência notável na variabilidade climática do Estado. Os resultados aqui apresentados sugerem que, embora o ENOS tenha um efeito importante na produtividade da soja no RS, outros padrões de teleconexão podem ser relevantes na variabilidade interanual da produtividade 
agrícola do Estado e devem ser levados em consideração em estudos futuros, especialmente em situações que o ENOS se encontra na sua fase fria ou em situação de neutralidade.

\section{Conclusões}

Os resultados aqui apresentados indicam a utilidade dos índices IOS e MEI para estimativas de produtividade de soja no Rio Grande do Sul sendo o IOS o índice que apresenta as maiores correlações para os três grupos.

Destaca-se que as correlações mais elevadas só são observadas em períodos nos quais a soja já foi plantada e encontra-se em estágio de florescimento e enchimento de grãos. Este fator evidencia a necessidade da inclusão de outros índices associados a padrões de teleconexão que possuam correlações significativas em maiores defasagens.

Evidencia-se que os resultados referentes a fase quente do fenômeno estão de acordo com estudos prévios e estão associados a elevação da produtividade na maior parte dos casos. No entanto, nota-se uma menor influência em relação as fases neutras e frias já que em apenas metade dos casos houve queda de produtividade. Este fator não havia sido ressaltado em estudos prévios.

\section{Referências}

Alberto, C. M., Streck, N. A., Heldwein, A. B., Buriol, G. A., Medeiros, S. L. P. (2006). Água no solo e rendimento do trigo, soja e milho associados ao El Niño Oscilação Sul. Pesquisa Agropecuária Brasileira, 41(7), 1067-1075.

Berlato, M. A., Fontana, D. C. (1999). Variabilidade interanual da precipitação pluvial e rendimento da soja no Rio Grande do Sul. Revista Brasileira de Agrometeorologia, 7(1), 119-125.

Berlato, M. A., Fontana, D. C. (2003). El Niño e La Niña: impactos no clima, na vegetação e na agricultura do Rio Grande do Sul; aplicações de previsões climáticas na agricultura. Editora UFRGS, Porto Alegre.

CEPEA (2017). PIB Agro CEPEA-USP/CNA. URL http: / / cepea.esalq.usp.br/pib/.

da Cunha, G. R., Barni, N. A., Haas, J. C., Maluf, J. R. T., Matzenauer, R., Pasinato, A., Pimentel, M. B. M., Pires, J. L. F. (2001). Zoneamento agrícola e época de semeadura para soja no Rio Grande do Sul. Revista Brasileira de Agrometeorologia, 9(3), 446-459.

Diaz, A. F., Studzinski, C. D., Mechoso, C. R. (1997). Relationships between Precipitation Anomalies in Uruguay and Southern Brazil and Sea Surface Temperature in the Pacific and Atlantic Oceans. Journal of Climate, 11, 251-271.

Fontana, D. C., Berlato, M. A., Lauschner, M. H., de Mello, R. W. (2001). Modelo de estimativa de rendimento de soja no Estado do Rio Grande do Sul. Pesquisa Agropecuária Brasileira, 36(3), 399-403.

Grimm, A. M., Ferraz, S. E. T., Gomes, J. (1998). Precipitation Anomalies in Southern Brazil Associated with El Niño and La Niña Events. Journal of Climatology, 11, 2863-2880.

Hoogenboom, G. (2000). Contribution of agrometeorology to the simulation of crop production and its applications. Agricultural and Forest Meteorology, 103, 137-157.

IBGE (2017). Produção Agrícola Municipal. URL https : / s sidra . ibge . gov . br/Tabela/1612.

Kaiano, M. T., Andreoli, R. V. (2007). Relations of South American summer rainfall variations with the Pacific Decadal Oscillation. International Journal of Climatology, 27, 531-540.

Kayano, M. T., Andreoli, R. T. (1998). Relations of South American summer rainfall interannual variations with the Pacific Decadal Oscillation. Journal of Climate, 11, 2863-2880.

Lazzari, M. R. (2006). Agropecuária gaúcha em 2005: estiagem, câmbio e crise. Indicadores Econômicos FEE, 33(4), 41-50.

Lizumi, T., Luo, J., Challinor, A. J., Sakurai, G., Yokozawa, M., Sakuma, H., Brown, M. E., Yagamata, T. (2014). Impacts of El Niño Souther Oscillation on the global yields of major crops. Nature Comunications, 5.

Rao, G. V., Hada, K. (1990). Characteristics of rainfall over Brazil annual variations and connections with the Southern Oscillation. Theoretical and Applied Climatology, 42, 81-91.

Reboita, M. S., Ambrizzi, T., da Rocha, R. M. (2009). Relationship between the Southern Annular Mode and Southern Hemisphere atmospheric systems. Revista Brasileira de Meteorologia, 24(1), 48-55. 
Reboita, M. S., Gan, M. A., da Rocha, R. M., Ambrizzi, T. (2010). Regimes de precipitação na América do Sul: uma revisão bibliográfica. Revista Brasileira de Meteorologia, 25(2), 185-204.

Santos, E. B., Diniz, G. B. (2014). Oceanic indices and their relations with the monthly precipitation in Rio Grande do Sul state, Brazil. Revista Brasileira de Geofísica, 32, 371-381.

Sentelhas, P. C., Battisti, R., adn J. R. B. Farias, G. M. S. C., Hampf, A. C., Nendel, C. (2015). The soybean yield gap in Brazil magnitude, causes and possible solution for sustainable production. Journal of Agricultural Science, 153, 1-18.

Ward, J. H. (1963). Hierarchical grouping to optimize an objective function. Journal of the American Statistical Association, $58(301), 236-244$.

Wenjiao, S., Fulu, T., Zhao, Z. (2013). A review on statistical modelos for identifiying climate contributions to crop yields. Journal of Geographical Sciences, 23, 567-576.

Wilks, D. S. (2006). Statistical Methods in the Atmospheric Sciences, vol 78, $2^{\circ}$ edn. Academic Press, Elsevier, San Diego, California, United States of America.

Zanon, A. J., Streck, N. A., Grassini, P. (2016). Climate and management factors influence soybean yield potential in a subtropical environment. Agronomy Journal, 108, 1447-1454.

Diogo Alessandro Arsego

Universidade Federal de Santa Maria, Santa Maria, RS, Brasil E-mail: diogo.arsego@gmail.com

Simone Erotildes Teleginski Ferraz

Universidade Federal de Santa Maria, Santa Maria, RS, Brasil E-mail: simonetfe@gmail.com

Nereu Augusto Streck

Universidade Federal de Santa Maria, Santa Maria, RS, Brasil E-mail: nstreck2@yahoo.com.br

Andrea de Oliveira Cardoso

Univesidade Federal do ABC, SP, Brasil E-mail: andreca@gmail.com

Alencar Junior Zanon

Universidade Federal de Santa Maria, Santa Maria, RS, Brasil E-mail: alencarzanon@hotmail.com 